\title{
Evaluating the international society for stem cell research's 2016 guidelines As they apply to clinical trials with therapies derived from pluripotent stem cells
}

\author{
Audrey R. Chapman* \\ Healey Professor of Medical Ethics and Humanities, Professor of Community Medicine, Chair UConn Stem Cell Research Oversight Committee, Farmington,
} CT, USA

\begin{abstract}
Human pluripotent stem cells hold significant promise for developing therapies for diseases and disorders for which there are no current treatments and for replacing, engineering, or regenerating human cells, tissues, and possibly even developing organs to restore or establish normal functioning. However, there are many scientific and ethical hurdles that researchers must address in order to proceed responsibly. Early clinical trials with innovative products often raise complex ethical challenges, particularly the first in human trials with novel therapies which most of the trials with therapeutics developed from pluripotent cells will be. This paper discusses and evaluates the 2016 Guidelines for Stem Cell Science and Clinical Translation issued by the International Society for Stem Cell Research (ISSCR) which is currently the only comprehensive set of ethical and scientific guidelines that provide guidance for pluripotent stem cell clinical trials.
\end{abstract}

\section{Paper Text}

Human pluripotent stem cells hold significant promise for developing therapies for diseases and disorders for which there are no current treatments. Pluripotent stem cells may also provide a basis for replacing, engineering, or regenerating human cells, tissues, and possibly even organs to restore or establish normal functioning. However there are many scientific and ethical hurdles that researchers must first address in order to proceed responsibly. One of these is conducting clinical trials. Early clinical trials with innovative products often raise complex ethical challenges, particularly first in human trials like most of the trials with therapeutics developed from pluripotent cells will be. This paper discusses and evaluates the 2016 Guidelines for Stem Cell Science and Clinical Translation issued by the International Society for Stem Cell Research (ISSCR) [1]. They are the first set of comprehensive guidelines for conducting pluripotent stem cell research and the first and currently the only ethical guidelines that deal with clinical trials. The Guidelines come at an opportune time as the pluripotent stem cell field is moving into early clinical trials. This paper will focus on the sections of the Guidelines that provide guidance for clinical trials. While there have been several articles published about the Guidelines, including several written by members of the drafting committee, they are mostly descriptive providing an overview of the topics covered [2]. None to date have provided an in-depth analysis of the sections dealing with clinical trials as this article will do. Moreover, in contrast with the previous articles published on the ISSCR Guidelines, this article will be a critical examination of both the contributions and limitations of those sections of the Guidelines.

\section{Background}

In 1998 stem cell investigators at the University of Wisconsin successfully isolated, cultured, and partially characterized human stem cells (hESCs) from the inner cell mass of early stage embryos at the blastocyst stage. Although the scientific promise of these cells was quickly grasped, hESC research also became ethically and politically controversial because the process for harvesting the cells results in the destruction of the embryo. Religious communities and people who accord the embryo a high moral status found this deeply problematic even though there are perhaps one million surplus embryos, most of which are unlikely to ever be used. In another landmark discovery in the stem cell field, in 2007 two teams of researchers were able to reprogram human skin cells into an earlier and undifferentiated state with many of the characteristics of hESCs. Importantly, the development of these reprogrammed or induced pluripotent stem cells (hiPSCs) did not require the destruction of human embryos. It has been unclear, however, whether hESC and hiPS cells are completely comparable biologically and whether they are clinically interchangeable. Some stem cell scientists have raised questions about whether hiPSCs are suitable for clinical applications. Some of these cautions will be noted later in the paper.

Despite the scientific promise and the ethical controversy surrounding research with pluripotent stem cells, the United States government has not issued authoritative guidelines for research, clinical trials, and/or the translation of pluripotent stem cells into therapies. To try to fill the vacuum, the National Academies of Sciences (NAS), an independent scientific body, sponsored an advisory committee funded by several foundations from 2005 until 2010 that drafted and then updated voluntary guidelines for the responsible practice of pluripotent stem cell research [3]. The Committee was disbanded in 2010 due to a lack of funding. Moreover, the NAS guidelines do not offer

Correspondence to: Audrey R. Chapman, Healey Professor of Medical Ethics and Humanities, Professor of Community Medicine, Chair UConn Stem Cell Research Oversight Committee, Farmington, 263 Farmington Ave CT, USA, Tel: 860679 1590; E-mail: achapman@uchc.edu

Received: April 01, 2018; Accepted: April 10, 2018; Published: April 13, 2018 
recommendations for how to conduct clinical trials with pluripotent stem cells. The Food and Drug Authority's (FDA) Cellular, Tissue, and Gene Therapies Advisory Committee (CTGTAC) did devote a 2008 meeting to clinical trials with therapies developed from human embryonic stem cells, but it decided it was then premature to develop guidelines [4]. In 2009 President Obama eliminated the restrictions President Bush had imposed on government funding of pluripotent stem cell research that limited eligibility to hESC stem cell lines created before August 2001 and asked the National Institutes of Health (NIH) to develop guidelines for federal funding. Although many in the stem cell community had hoped the new guidelines would address a broad range of research issues as well as funding, the $2009 \mathrm{NIH}$ guidelines for Human Stem Cell Research focus on the policies and procedures for research projects using hESC lines to be eligible for federal funding and do not consider broader issues of research and applications [5]. Additionally, the United States does not have a national body mandated to provide oversight for pluripotent stem cell research that can offer the kind of constructive guidance the Recombinant DNA Advisory Committee (RAC) has provided for gene transfer research and trials.

Thus the ISSCR Guidelines fill a need and come at an opportune point in time since clinical trials with therapeutics developed from pluripotent stem cells are beginning. A 2015 survey using the Clinical Trials.gov database, the International Clinical Trials Registry Platform, PubMed, press releases, and relevant websites of companies and institutions identified eight ongoing clinical trials with therapeutics developed from the two primary types of pluripotent stem cells. The trials, primarily Phase I initiatives with small numbers of patients to test safety and dosage levels, are testing potential therapies to treat spinal cord injury, macular degeneration, a type 1 diabetes an immunotherapy vaccine for lung cancer, and heart failure [6]. In 2015, the California Institute for Regenerative Medicine (CIRM) launched the Alpha Stem Cell Clinics, a network of six leading medical centers in California, to accelerate the development of stem cell treatments. The CIRM website (as of October 2017) lists 41 ongoing trials it has funded, many of them potential therapies developed from pluripotent stem cells [7]. In addition, in June 2017 scientists in China announced they were about to start clinical trials with therapies derived from hESCs to treat vision loss and Parkinson's disease [8].

As noted, early clinical trials with innovative mechanisms and therapies often raise ethical challenges. The level of risk to participants in FIH trials often correlates with the innovativeness of the therapy under consideration and pluripotent stem cell derived therapies qualify as novel and high-risk products. The ethical appropriateness of clinical research requires having a favorable risk-to-benefit ratio and protecting patients from excessive risk, but these standards are difficult to achieve in FIH trials with novel therapies which involve the greatest degree of uncertainty at any point in the therapeutic development process [9]. In contrast with later stage trials, Phase I trials have little or no human experience on which to draw when conducting a risk-benefit analysis [10]. Extrapolating from laboratory and animal studies to humans is a complex process under all circumstances, but even more so in FIH trials which usually lack data from comparator studies in humans to help guide the process. Given the limitations of animal models and the differences between human and animal physiology, toxicological studies in animals may be poor predictors of human outcomes [11]. And notwithstanding the decision of the FDA to authorize several Phase I clinical trials with a hESC-derived therapeutic, the understanding of the scientific mechanism of action of pluripotent stem cells is still limited and few safety questions regarding human applications have been thoroughly addressed in animal research.
For these reasons the 2016 Guidelines for Stem Cell Science and Clinical Translation issued by the International Society for Stem Cell Research (ISSCR) [12] represent an important contribution to the field. Founded in 2002 to provide a forum for communication and education in the emerging field of stem cell research and regenerative medicine, ISSCR is the largest international professional organization engaged with stem cell research. ISSCR previously developed Guidelines for the Conduct of Human Embryonic Stem Cell Research in 2006 [13] and Guidelines for the Clinical Translation of Stem Cells in 2008 [14], but the stem cell field has advanced since those guidelines were drafted and in the process encountered new ethical, social, and policy challenges. Also the earlier guidelines did not address the process of conducting clinical trials. The 2016 Guidelines were developed by an international task force of 25 leading stem cell scientists and ethicists with extensive experience with stem cells. Draft guidelines were posted for a three month period of public comment to encourage review and input by others in the stem cell community before they were finalized. The taskforce also sought perspectives from individuals within regulatory authorities, funding agencies, industry, patient advocacy organizations, and professional societies [15].

\section{Fundamental Ethical Principles}

The ISSCR's Guidelines build on widely shared ethical principles in science, research with human subjects, and medicine. Some of the ethical principles and the guidelines following from them would apply to any basic research and clinical translation initiative and others address challenges that are especially relevant for stem cell-based research. The Guidelines identify five guiding principles: the integrity of the research enterprise, the primacy of patient welfare, respect for research participants, the need for transparency, and the importance of social justice. According to the Guidelines, the primary goals of stem cell research are to advance scientific understanding and to generate evidence for addressing medical and public health needs. To assure the integrity of the research enterprise so as to maintain public confidence and ensure the data will be reliable and accessible, research should be overseen by qualified investigators through independent peer review and oversight, replication, and accountability at each stage of research. The primacy of patient welfare means that physicians and researchers owe primary duty to the patient and/or research subject and therefore must never unduly place vulnerable patients or subjects at risk. Respect for research participants requires that human subjects can exercise valid informed consent when they have adequate decisionmaking capacity. Transparency requires researchers to make accurate scientific information available to interested parties including patient communities [16].

The guideline on social justice is particularly notable because few other treatments of the translation of scientific discoveries include a discussion of social justice. Moreover there has been a fundamental inconsistency in the rationale of the state programs that have played a major role in the funding of pluripotent stem cell research which have both argued that this research will benefit patients by discovering new cures and at the same time that the state will reap a financial profit from its investment. It is standard practice for most of the state funders to promote patenting rather than open licensing of discoveries and to have a clause in their funding contracts requiring royalty payments to the state [17].

According to the Guidelines, the benefits of clinical translation efforts should be distributed justly and globally, with particular emphasis on addressing unmet medical and public health needs. To accomplish 
this goal, the guidelines recommend that advantaged populations should seek to share benefits with disadvantaged populations and that trials should strive to enroll populations diverse in age, sex, and ethnicity. Like other proponents of inclusiveness, the Guidelines state that social justice requires that trials should strive to include women as well as men, and members of racial and ethnic minorities. They specifically caution that burdens associated with clinical translation should not be borne by populations who are unlikely to benefit from the knowledge produced in these efforts. In addition, new products should not be approved for routine clinical use without a favorable balance of risk and benefit being shown in trial. The Guideline also stress that developers should strive to maximize access to treatments [18]. Later in the discussion of access and economics the Guidelines affirm that research, clinical, and commercial activities should seek to maximize affordability and accessibility [19]. However the Guidelines do not go so far as to recommend that publicly funded research should require that products developed from the stem cell research be available on an affordable basis to disadvantaged patient populations.

Fluid and umbilical cord blood, raise additional questions about which type of stem cell is appropriate to use in particular types of research, particularly in efforts to develop clinical therapies. There are both ethical and scientific implications in the choice of which type of pluripotent or multipotent stem cell to use. However the Guidelines do not address this critical issue. Even if the drafters of the Guidelines did not want to offer recommendations, the Guidelines could have offered criteria for researchers to apply in making a decision.

The failure to do so is particularly problematic because the field currently favors research with hiPSCs, most likely to avoid the ethical controversies that hESCs can potentially entail and the anticipation that funding opportunities for research with hiPSCs will be greater, if not now, in the future. However, hESCs represent the gold standard for the field. Almost all the clinical trials to-date have been of hESC-derived therapies. Moreover hiPSCs have been shown to have problems that may limit their potential as the basis for clinical therapies. To mention a few issues, hiPSCs have been shown to have far more abnormalities than hESC derivatives [20]. The reprogramming process and subsequent culture of hiPSCs in vitro induce genetic and epigenetic abnormalities in these cells that affect their safe use [21]. In contrast with the embryonic cells from which hESCs are derived, the somatic cells that are being regressed to obtain hiPSCs have accumulated mutations from the aging process that are then transferred to the hiPSCs.

\section{Cell Processing and Manufacture}

Current regulatory standards attempt to ensure that investigational products prove to be safe, effective, and of sufficient quality for their intended medical use in humans before their clinical application. Cellular derivatives present unique challenges in their processing and manufacture in all three categories. Stem cells present greater problems for quality control in their processing and manufacture than small molecule based medical products. As the Guidelines point out, cells in culture age and may accumulate genetic and epigenetic changes that affect their differentiation behavior and function. Pluripotent stem cells have additional risks due to their pluripotency which makes them prone to acquire mutations when maintained for prolonged periods in culture, to differentiate into inappropriate cellular phenotypes, to form benign teratomas and sometimes malignant outgrowths as well, and to fail to mature. Scientific understanding of how to control these processes is still evolving [22]. Additionally the unique characteristics of pluripotent stem cells raise new concerns about the identity, purity, potency, and viability of the pluripotent stem cell derivatives in therapeutic products. Also compared with pharmaceuticals, biological, and somatic cellular therapies pluripotent stem cell-base products have a distinctive dynamic heterogeneity that increases uncertainty and safety risks [23]. Moreover, as the Guidelines note, even minimal manipulation of cells outside the human body introduces additional risks of contamination with pathogens. Also cells in culture age and may accumulate genomic and epigenomic mutations that could lead to altered cell function or malignancy [24]. The manufacturing side presents additional challenges related to quality control, scalability, sustainability, and the costs of the goods [25].

The Guidelines offer a series of recommendations to at least begin to address these issues. According to the Guidelines, GMP (Good Manufacturing Practices) procedures should be followed for extensively manipulated stem cells, such as pluripotent stem cells, when they are intended for clinical application. Specifically, the Guidelines state that the degree of oversight and review of cell processing and manufacturing protocols should be proportionate to the risk induced by manipulation of the cell, their source and intended use, the nature of the clinical trial, and the number of research subjects who will be exposed to them [26], but these data may not be available, particularly in early phase clinical trials. The Guidelines also recommend that risks for tumorigenecity be rigorously assessed for any stem cell-based product, particularly for pluripotent stem cells [27]. Another recommendation is that the stringency of review for cell processing and manufacture should increase as cells are tested in later phase clinical trials, used in practice settings, or administered to multiple patients [28].

\section{Preclinical Studies: The Use of Animal Models}

The selection of appropriate animal models is a key element for preclinical studies. The Guidelines discussion of animal models is divided into a number of sections of the document. The consideration of animal welfare in the Guidelines notes that researchers should adhere to the principles of the three Rs: to reduce numbers, refine protocols, and replace animals with in vitro or non-animal experimental platforms whenever possible. The Guidelines also state that researchers should select animal models that best match the human disease for which treatment is sought but do not offer criteria to assist in making that determination. Additionally, they recommend that researchers and sponsor should ensure effects in animals are robust by replicating findings, ideally in more than one animal model [29]. The section in the Guidelines on biodistribution studies notes that while rodents or other small animal models are typically a necessary step in the development of stem cell-based therapies they are likely to reveal only major toxic events. The Guidelines also observe that the similarity of many crucial physiological functions between large mammals and humans may favor testing in at least one large animal model [30]. This is an important point because to-date preclinical pluripotent stem cell research has generally relied predominantly on rodent models even when rodents seem to be an inappropriate animal model. Additionally the Guidelines discuss animal studies in the section on efficacy studies proposing that small animal models be used to assess the biological mechanisms of activity and to optimize implementation of an intervention. Again, the Guidelines advise that large animal studies are believed to better emulate human anatomy or pathology and are preferable where risks to human subjects in anticipated clinical trials are high as they often will be in early clinical trials with an innovative therapy. The Guidelines are appropriately cautious about using non-human primates suggesting that decisions be made on a case-by-case basis and only where nonhuman primates are anticipated to be able to provide information not 
obtainable with other models [31]. Many of the points made in the Guidelines parallel the discussion at the 2008 meeting of the FDA's Cellular, Tissue, and Gene Therapies Advisory Committee (CTGTAC) considering clinical trials with therapies developed from hESCs but the members of the CTGAC were more definitive about the importance about the need to use large animal models [32]. I think it would have been preferable for the Guidelines to be more prescriptive about the need to use large animal studies.

\section{Preclinical Studies}

In contrast with later phases of clinical trials, for which there are human subject data available, the justification for beginning Phase I clinical trials of an investigational agent relies entirely on the quality and efficacy of the preclinical evidence. The decision to go forward with FIH trials for which there are not comparator studies rests on the foundation of the appropriateness and quality of preclinical research. However there is a growing literature documenting problems with the preclinical data used to support the initiation of Phase I trials. Deficiencies noted include inadequate measures to control bias, absence of measures for random treatment allocation and blinded outcome assessment in the animal studies, and failure to account for missing data. Yet another issue is that some FIH trials are initiated before preclinical data have received adequate peer review. Financial incentives may also contribute to premature entry into clinical trials [33]. Preclinical literature also shows evidence of publication bias, that is, neutral or negative animal studies may be more likely to remain unpublished than successful studies [34]. In addition, the designs of some clinical trials have failed to take into account the limitations of efficacy observed in animal data as well as discounting the risks.

To address these issues, the Guidelines seek to articulate high standards for preclinical design and study reporting. According to the Guidelines, before beginning clinical studies with stem cells in humans, researcher should have rigorous evidence of safety and efficacy in preclinical research with in vitro studies and/or animal research. Preclinical studies must be rigorously designed, reported, reviewed independently, and subject to regulatory oversight. The Guidelines further underscore that the strength of the preclinical evidence required for trial launch should be proportionate with the risks, burdens, and ethical sensitivities of the anticipated trial [35]. The Guidelines also recommend that more stringent design and reporting standards should be demanded when planned trials involve human subjects with less advance disease; when invasive delivery approaches are anticipated; or in cases where the cell product involves greater risk and uncertainty [36]. Other considerations addressed are that studies designed to inform trial initiation should have high internal validity and researchers should reduce bias and random variation by ensuring their studies have adequate statistical power [37].

Despite noting that the Declaration of Helsinki and the Nuremberg Code strongly encourage undertaking animal studies prior to clinical trials, the Guidelines appear to contemplate possibly moving forward to human trials at least in some situations solely on the basis of in vitro studies [38]. This is problematic because stem cells may behave very differently when transplanted into bodies than they do in a dish. Nor is it possible to assess whether and where they are likely to migrate once placed in a body. The Guidelines do note that cells can change after transplantation in unpredictably ways [39]. The Guidelines appear to try to justify bypassing animal testing in part by downgrading the usefulness of animal models. The document notes that extrapolating from animal models to humans is more challenging for cell therapies than for small molecule products [40]. Even if this is the case, it does not warrant discarding the animal research that has been the gold standard of preclinical studies. Instead, it calls for conducting research on a variety of animal models so as to assess the likely impact of transplanting the cells into research subjects, and elsewhere in the document the Guidelines do suggest such.

\section{Initiation of Clinical Trials}

While the challenges of conducting first-in-human trials ethically and responsibly suggest the need for caution in moving from the preclinical to the clinical phase of research, there are often countervailing pressures to begin pluripotent stem cell trials. Human stem cell research is widely identified as holding enormous potential for medical research and the development of treatments for a wide range of diseases and medical conditions for which adequate therapies do not exist. The publicity surrounding developments in the field appears to have led many patients and patient support groups to view prospective stem cell-based therapies as potential miracle cures and to clamor for their development and availability. Additionally, the competitive spirit of researchers and their academic institutions can incline them to move forward as quickly as possible to develop breakthrough discoveries and therapies, particularly in situations where there are opportunities for commercialization. Also much of the early initiatives for the development of pluripotent stem cell research came from small biotechnology companies, and their funding prospects and financial viability have often depended on their ability to bring a product into clinical trials. States which have provided funding for stem cell research have also been keen to show benefit to the public for their investment. For example, the California Institute of Regenerative Medicine (CIRM) has been under pressure to show benefits for the investment of the $\$ 3$ billion it received from a referendum that led to the sale of state bonds to underwrite the cost of stem cell research, and for this reason it established a mechanism to accelerate the process of bringing research into clinical trials.

The ISSCR Guidelines recognize these pressures and the problematic trend toward initiation of clinical applications and trials far in advance of what is warranted by sound and rigorously assessed preclinical research. Seeking to balance risk with opportunity, the Guidelines caution against moving to clinical trials and applications until there is sound, rigorous, and dispassionately assessed preclinical evidence with a high probability of success [41].

\section{Standards for Clinical Research Conduct}

The briefing document prepared for the 2008 meeting of the FDA's Cellular, Tissue, and Gene Therapies Advisory Committee noted that early-phase clinical trials of all cell therapies expose participants to potential risks that differ substantially from those associated with Phase I drug trials: their pharmacological disposition is unpredictable; unchecked proliferation is a real possibility in cellular products; and the surgical procedures required for administration of many cellular products may pose additional risks. One such issue the document discusses is the intrinsic capacity of hESCs to generate teratomas or tumors. The Guidelines recognize many of these problems and additionally note that cell therapy products derived from hESCs will be heterogeneous in their composition and may contain residual undifferentiated hESCs and partially differentiated cells with the capacity to proliferate and differentiate further. It explains that these cells will then have the ability to migrate from their target site of administration and possibly undergo inappropriate differentiation at a non-target site. 
For these reasons, according to the Guidelines, early phase clinical trials of hESCS-derived cellular products will have to be designed carefully in order to ensure the safety of enrolled subjects and data supporting a reasonable possibility of efficacy may need to be particularly strong [42]. The Guidelines also recommend that researchers conduct initial tests of a novel strategy under lower risk conditions before proceeding to higher risk study conditions even if the latter are more likely to confer therapeutic benefit. This would entail beginning clinical trials at lower doses, using less aggressive co-interventions, and staggering testing before expanding the trial to additional subjects. Possibly in response to the criticisms of the Geron Company's first-in-human trial stem cell trial [43], which used research subjects who had suffered very recent severe spinal injuries (less than two weeks before beginning the trial), the Guidelines state that researchers should, in general, validate safety and techniques with advanced disease subjects before testing their products in research subjects with more recent disease onset [44].

Safety will be a significant factor in evaluating the appropriateness of clinical applications of pluripotent stem cell derivatives, particularly whether hiPSC derivatives can meet the quality, stability, and purity standards required for human medical use. Both hESC and hiPSC derivatives have been shown to have genetic aberrations [45]. It is here relevant to note that hiPSC derivatives appear to have more serious problems and a greater number of abnormalities than hESC derivatives, some likely to be from the adult cells from which they are developed and others from the regression process and from the time spent in culture. A study comparing hESCs with hiPSCs showed that induced stem cells were not of the same quality as embryonic stem cells and tended to age and die earlier than their embryonic stem cell counterparts [46]. Additionally, hiPSCs have been shown to retain an epigenetic memory and residual characteristics of the somatic cells from which they are derived and to fail to reprogram cell methylation patterns [47]. A 2011 review of the scientific literature titled "the dark side of induced pluripotency" concluded that the reprogramming process and subsequent culture of hiPSCs in vitro induce genetic and epigenetic abnormalities in these cells that are likely to affect their safe use [48]. George Daley, a leading stem cell scientist, has predicted that it will take decades to figure out the principles to make hiPSCs deliverable as medicines [49]. Other analysts have attributed the failure to date of stem cell therapies to meet therapeutic expectations to the role of stem cell ethics in influencing the selection of clinically unfit somatic-based cells over embryonic stem cells: "By sidelining the cells with the greatest growth capacity and plasticity on the basis of moral objections, the regenerative potential of stem is left unfilled. It is this choice, above all, which has limited the application of stem cells in medicine [50]." The Guidelines do not address this important issue.

\section{Informed Consent Issues}

Voluntary and informed consent of subjects participating in a scientific study is a central international principle of research ethics and an expression of respect for human persons. Informed consent is identified as fundamental in stem cell research by the U.S. National Academy of Sciences [51] and the California Institute for Regenerative Medicine (CIRM) [52]. Informed consent considerations also play a major role in the NIH guidelines evaluating whether specific hESC stem cell lines are eligible to be used with research funding from the U.S. government [53]. The Guidelines discuss informed consent issues related both to the donation of cells for allogeneic applications [54] and to participants in stem cell research [55].
Concerns have been expressed about the possibilities of obtaining meaningful consent in early phase clinical trials with pluripotent stem cells. As I have noted elsewhere, the informed consent process in early phase trials requires that potential subjects be accurately informed of the purpose, methods, risks of adverse events, and the very limited, if any, prospect of therapeutic benefits; that they understand this information and be able to apply it to their own situation; and that they make a voluntary and uncoerced decision as to whether to participate in the trial [56]. Each of these components can be especially problematic for FIH trials since there is often no reliable information about benefits and risks for studies of agents never before used in humans. Directors of clinical trials and IRBs reviewing and evaluating informed consent documents have the unenviable task of encouraging potential subjects to participate in the trial while dissuading them of the "therapeutic misconception" that confuses scientific research with therapy. Communicating uncertainty and risk and determining whether a patient understands the information is difficult. It is made more so because volunteers entering clinical trials often overlook discussions of risk and focus their attention on the possible benefits.

Importantly, the Guidelines recognize that early phase trials involving stem cell-based interventions may enroll research subjects who have exhausted standard treatment options and therefore may be prone to overestimating the likelihood of receiving benefit. They underscore that consent procedures in early phase trials of stem cellbased interventions should therefore work to dispel potential research subjects' overestimation of benefit and therapeutic misconception. To do so the Guidelines recommend the following procedures: (1) conducting informed consent discussions with a discussant present who is independent of the research team; (2) explaining the rarity of major therapeutic benefits in early phase subjects to prospective subjects; (3) testing the comprehension of prospective subjects before accepting their consent; (4) avoiding the use of language that has therapeutic connotations; and (5) supplementing consent forms with additional educational materials [57]. Additionally, it would be important for researchers and sponsors of the research and trials to refrain from exaggerating the likelihood of a therapeutic result from early clinical trials in their communications with the media. This hype surrounding stem cell research plays an important role in promoting unrealistic expectations.

\section{Oversight of Trials}

According to the Guidelines, the overarching goal of research oversight "is to ensure that a research study will likely be safe, protect human subjects, and have scientific and medical merit, and that it is designed and carried out in a manner that will yield credible data and enhance scientific and medical understanding [58].” The Guidelines recognize that accomplishing these goals requires that all research involving clinical applications of stem cell-based interventions must be subject to prospective review, approval, and ongoing monitoring by independent human subject review committees composed of members with appropriate scientific, medical, and ethical expertise. The independent experts should be competent to evaluate the preclinical studies that form the basis for proceeding to a trial and the design of the trial. In addition, peer review should assess whether the proposed clinical trial is likely to lead to important new knowledge or an improvement in health [59].

\section{Choice of Comparators}

According to the Guidelines, clinical research should compare new stem cell-based interventions with the best currently available 
therapeutic approaches that could reasonably be expected to be available to the local population at the site of testing. If there are no proven effective treatments for a medical condition and the stem-cell based interventions involve invasive delivery, the Guidelines suggest that it may be appropriate to test them against a placebo or sham comparators. The Guidelines also caution against conducting trials in a foreign country if the goal is to solely benefit patients in the home country of the sponsoring agency. As in trials of small moleculebased therapeutics, the test therapy, if approved, should realistically be expected to become available to the population in the locale participating in the clinical trial as well.

\section{Objectives of Trials}

The Guidelines offer a high standard for clinical research to be warranted. The text recommends that a proposed stem cell-based intervention must aim at being clinically competent with or superior to existing therapies or able to meet a unique therapeutic demand [60].

\section{Long-term safety studies}

In contrast with small molecule clinical trials that can be halted if problems occur, stem cells once implanted cannot be removed from trial subjects. For better or worse the transplanted stem cells will have longterm effects. In view of the likelihood of the long term persistence of the cells and the irreversibility of at least some cell-based interventions, the Guidelines call for long-term safety studies in animals and longterm follow-up in patients. However, the Guidelines do not define what long-term means. Instead it states that length of follow up should vary with the survival expectancy for patient populations projected for study enrolment [61]. It would have been helpful to be more specific. Given that many trials will likely be halted early, it would have also been helpful to underscore that these researchers and companies bear the same ethical responsibility for long-term monitoring.

\section{Transparency and Reporting of Research Results}

The Guidelines recommend that all trials should be prospectively registered in public data bases to provide a mechanism for promoting unbiased reporting of trials. The registration of trials also would promote access to patients who might not otherwise be able to be informed about them. Recognizing that many studies fail to report adverse events, the Guidelines direct investigators to report adverse events including their severity and their potential relationship to the candidate therapy. Also the Guidelines strongly encourage researchers to promptly publish all clinical trials research results regardless of whether the outcomes are positive, negative, or inconclusive [62].

\section{Conclusion}

The 2016 ISSCR Guidelines represent an important development for the stem cell field and provide an authoritative standard for the conduct clinical trials. Hopefully the Guidelines will be widely adopted for clinical trial guidance despite their lack of official status. Hopefully at least some of the omissions noted in this paper will be addressed in a future update.

\section{References}

1. ISSCR (2016) Guidelines for Stem Cell Science and Clinical Translation. International Society for Stem Cell Research pp: 1-37.

2. See for example, Daley, G Q, Hyun I, Apperley, J F, Barker, R A, et al., (2016) Setting Global Standards for Stem Cell Research and Clinical Translation: The 2016 ISSCR Guidelines. Stem Cell Reports 6: 787-797.
3. Guidelines for Human Embryonic Stem Cell Research (2005) Human Embryonic Stem Cell Research Advisory Committee, National Research Council and Institute of Medicine of the National Academies. Washington: D.C.

4. U.S. Food and Drug Administration (2008) Cellular, Tissue, and Gene Therapies Advisory Committee. Cellular Therapies Derived from Human Embryonic Stem Cells - Considerations, Meeting Minutes.

5. NIH (2009) National Institutes of Health Guidelines for Human Stem Cell Research, National Institutes of Health.

6. Ilic D, Devito L, Miere C, Codognotto S (2015) Human embryonic and induced pluripotent stem cells in clinical trials. Br Med Bull 116: 19-27. [Crossref]

7. Nolan RP, Payne AY, Ross H, White M, D'Antono B, et al. (2014) An Internet-Based Counseling Intervention With Email Reminders that Promotes Self-Care in Adults With Chronic Heart Failure: Randomized Controlled Trial Protocol. JMIR Res Protoc 3: e5. [Crossref]

8. Cyranoski D (2017) Trials of embryonic stem cells to launch in China. Nature 546 15-16. [Crossref]

9. Kimmelman J, London AJ (2011) Predicting Harms and Benefits in Translational Trials: Ethics, Evidence, and Uncertainty. PLOS Medicine 8: e1001010.

10. Kimmelman J (2010) Gene Transfer and the Ethics of First-in-Human Research: Lost in translation. New York p. 3.

11. Chapman AR (2011) Addressing the Ethical Challenges of First-in-Human Trials. $J$ Clinic Res Bioeth 2: 113.

12. International Society for Stem Cell Research (2015) Guidelines for Stem Cell Science and Clinical Translation.

13. International Society for Stem Cell Research (ISSCR) (2006) Guidelines for the Conduct of Human Embryonic Stem Cell Research.

14. International Society for Stem Cell Research (ISSCR) (2008) Guidelines for the Clinical Translation of Stem Cells

15. ISSCR Guidelines (2016) Preface.

16. ISSCR Guidelines (2016) section 1: 5.

17. https://www.google.com/search?q=Maryland+stem+cell+research+fund\&rlz=ICHBUS748\&oq=Maryland..+

18. Kimmelman J, Heslop HH, Sugarment J, Studer L, Benvenisty N, et al, (2016) Working group's discussion of the ethical principles in New ISSCR guidelines, clinical translation of stem cell research. ISSCR Guidelines 1: 5 .

19. ISSCR Guidelines (2016) section 3.5.2

20. Feng Q, Lu S, Klimanskaya I, Gomes I (2010) Hemangioblastic Derivatives from Human Induced Pluripotent Stem Cells Exhibit Limited Expansion and Early Senescence. Stem Cells 28: 704-712.

21. Pera MF (2011) Stem cells: The dark side of induced pluripotency. Nature 471: 46-47. [Crossref]

22. ISSCR Guidelines (2016) Section 3.1.2

23. Barazzetti G, Hyrst SA, Mauron A (2016) Adapting Preclinical Benchmarks for Firstin-Human Trials of Human Embryonic Stem Cell-Based Therapies. Stem Cells Transl Med 5: 1058-1066.

24. ISSCR Guidelines (2016) section 3.1 p. 13.

25. Markowitz-Shulman A, Addie S, Hackmann M (2017) Navigating the Manufacturing Process and Ensuring the Quality of Regenerative Medicine Therapies: Proceedings of a Workshop, Washington, D.C.

26. ISSCR Guidelines (2016) section 3.1.2 p. 14.

27. ISSCR Guidelines (2016), Section 3.2.2.2 p. 17.

28. ISSCR Guidelines (2016) section 3.1.2 pp. 14-15.

29. ISSCR Guidelines (2016) section 3.2.1 p. 16.

30. ISSCR Guidelines (2016), section3.2.2.1 p. 17.

31. ISSCR Guidelines (2016), section 3.2.3 p. 18.

32. U.S. Food and Drug Administration (2008) Cellular, Tissue, and Gene Therapies Advisory Committee. Cellular therapies derived from human embryonic stem cells Considerations for pre-clinical safety testing and patient monitoring. 
33. Kimmelman J (2010) Gene Transfer and the Ethics of First-in-Human Research: Lost in translation. New York p. 123.

34. Van der Worp HB, Howells DW, Sena ES, Porritt MJ (2010) Can Animal Models of Disease Reliably Inform Human Studies? PLOS Medicine 7: e00245.

35. ISSCR Guidelines (2016) section 3.2.1, p. 16.

36. ISSCR Guidelines (2016), section 3.2.1, p.16.

37. ISSCR Guidelines (2016), section 3.2.1.3, p. 16.

38. ISSCR Guidelines (2016), section 3.2, p. 15.

39. ISSCR Guidelines (2016), section 3.2, pp. 15-16.

40. ISSCR Guidelines (2016), section 3.2, pp. 15-16

41. ISSCR Guidelines (2016), section 3, p.13.

42. ISSCR Guidelines (2016), section 3, p.13.

43. Chapman AR, Scala C (2012) Evaluating the First-in-Human Clinical Trial of a Human Embryonic-Based Clinical Therapy. Kennedy Institute of Ethics Journal 3: 243-261.

44. ISSCR Guidelines (2016) section 3.3.3, p. 22.

45. Laurent LC, Ulitsky I, Slavin I (2011) Dynamic Changes in the Copy Number of Pluripotency and Cell Proliferations of Genes in Human ESCs and iPSCs during Reprogramming and Time in Culture. Cell Stem Cell 8: 1060118.

46. Feng Q, Lu S, Klimanskaya I, Gomes I (2010) Hemangioblastic Derivatives from Human Induced Pluripotent Stem Cells Exhibit Limited Expansion and Early Senescence. Stem Cells 28: 704-712.

47. Vaskova EA, Stekleneva AE, Medvedev SP, Zakian SM (2013) "Epigenetic Memory" Phenomenon in Inducted :luripotent Stem Cells. Acta Naturae 4: 15-21.
48. Pera MF (2011) Stem cells: The dark side of induced pluripotency. Nature 471: 46-47 [Crossref]

49. Garber K (2013) Inducing translation. Nat Biotechnol 31: 483-486. [Crossref]

50. Poulos J (2018) The limited application of stem cells in medicine: a review. Stem Cell Res Ther 9: 1. [Crossref]

51. Human Embryonic Stem Cell Research Advisory Committee (2005) National Academy of Sciences. 2005. Guidelines for human embryonic stem cell research. The National Academies Press, Washington, D.C pp. 88-91.

52. California Institute for Regenerative Medicine (2006) CIRM MES Regulations Title 17, California Code of Regulations Section p. 8

53. NIH (2009) National Institutes of Health. Guidelines on Stem Cell Research.

54. ISSCR Guidelines (2016) guideline 3.1.1.1, p. 14.

55. ISSCR Guidelines (2016) guideline 3.3.2, p. 21.

56. Chapman AR (2011) Addressing the Ethical Challenges of First-in-Human Trials, Clinical Research and Bioethics 2: 4 .

57. ISSCR Guidelines (2016) 3.3.3., p. 22.

58. ISSCR Guidelines (2016) 3.3.1, p. 19.

59. ISSCR Guideline (2016) 3.3.2, p. 20.

60. ISSCR Guidelines (2016) 3.2.2, p. 17.

61. ISSCR Guidelines (2016) 3.3.71.

62. ISSCR Guidelines (2016) 3.3.6, p. 24.

Copyright: $@ 2018$ Chapman AR. This is an open-access article distributed under the terms of the Creative Commons Attribution License, which permits unrestricted use, distribution, and reproduction in any medium, provided the original author and source are credited. 